$\xi=-1$

\title{
Finding the shortest path using the ant colony optimization
}

\author{
K. Yella Swamy ${ }^{1}$, Saranya Gogineni ${ }^{2}$, Yaswanth Gunturu ${ }^{2}$. Deepchand Gudapati ${ }^{2}$, Ramu Tirumalasetti ${ }^{2}$ \\ ${ }^{1}$ Assistant professor at Koneru Lakshmaiah Education Foundation \\ ${ }^{2}$ Student at Koneru Lakshmaiah Education Foundation \\ *Corresponding author E-mail: deepchand672@gmail.com
}

\begin{abstract}
An ant colony optimization(ACO) is a technique which is recently introduced , and it is applied to solve several np-hard problems ,we can get optimal solution in a short time Main concept of the ACO is based on the behavior of ants in their colony for finding a source of food. They will communicate indirectly through pheromone trails. Computer based simulation is can generate good solution by using artificial ants, by using that general behavior we are solving travelling Sale man problem.
\end{abstract}

Keywords: Optimization; Quadratic; Pheromone; Trails; NP-Hard.

\section{Introduction}

ACO is basically a technique to calculate optimized path between nest to food when ant looks for food going from their nest to food source the first ant goes from nest to food when it finds food while returning to nest it leaves pheromone trails(pheromone is a chemical substance which by ants to communicate indirectly to other ants)second ant sees the pheromone and goes in the that direction ,if there is more pheromone trails in path it feels that is the shortest path and goes in that direction if pheromones evaporated there will be no trail such that it is long path for finding the food .In ants there is 10-20 degrees of pheromone in their body some of the species doesn't have eyes they have sensing unit called decimal sensing unit with the help sensing unit they will find shortest path.

\section{Source of inspiration}

Each ant starts its from nest to find the food In different directions after some time some ants Will find food.While returning to home they will Deposit pheromone [1], [4] on the ground after some when Another goes for finding the food they will check for Pheromone path because if the pheromone is laid on Ground It is the shortest path if the path is long pheromone Will evaporate. To locate a briefest way, ants lay some pheromone on the ground, so a subterranean insect finds a another trail and choose to tail it. Thus, the aggregate conduct that develops as positive result where in the similar way the remaining ants chose a path where majority of ants move

\section{Brief analysis}

$\sum \quad$ Real ants follow a path from nest to food source.

$\sum \quad$ If any path deviation occurred in path or ant obstacle

based on the probability ants goes in different paths

$\sum \quad$ Pheromones are deposited on shortest path

$\sum \quad$ Ants will follow that pheromone path to get food.

\section{Literature survey}

Jinhui Yang about ACO for generalized TSP. He proposed a new method for ant colony optimization which is extended for the generalized travelling salesman problem. He used the concept of mutation to avoid locking into the local minima. Xiaohu Shi, Maurizio Marchese, and Yanchun Liang proposed this work. Zalilah Abd Aziz proposed a work about Ant colony hyper Heuristics for travelling salesman problem. In this paper, he developed an ACO with hyper-heuristics that finds a general way to solve TSP. He used two different types of searches for updating of pheromones. This is a simple way to implement the low level heuristics.

Michalis Mavrovouniotis about ACO with local search for dynamic TSP. In this work, a solution is found for the DTSP (dynamic TSP), i.e, a TSP which my be symmetric or asymmetric. An ACO with an US local search operator is introduced to solve DTSP's. Felipe M.Muller, and, Shengxiang Yang proposed this work.Hassan Ismkhan proposed a paper work about using Effective heuristics for ACO to handle large scale problems. In this paper, he pointed out some drawbacks of the current ACO's, which reduces the time and increases the space complexity in large scale problems. He used some effective heuristics and introduced strategies to solve the drawbacks.

Srikanta Patnaik proposed a work about Route optimization by Ant Colony Optimization technique.In this paper, he applied an ACO algorithm to find the available shortest path for TSP. He concluded that with good parameter combinations will increase route optimization.

Mateusz Sekara about multi-pheromone ant colony optim zation. In this work, socio-cognitive relations help the most. It has the usage of multiple pheromone values as given in the title. Michal Kowalski, Aleksandar Byrski, Bipin Indurkhya, and Dana Samson proposed this work.Chi-Bin Cheng and Chun-Pin Mao proposed a work on a modified ant colony system for solving the travelling salesman problem with time windows. In this paper, a time window (travel time of salesman) is solved. Two local heuristics are formulated to manage the travelling time of salesman in TSP. At each trip, the time is reduced to it's best. 
Viktor Danchuk about an improvement in ACO algorithm for optimizing a transport route with regard to traffic flow. In this paper, the ACO is modified to find the best possible delivery route with less traffic flow (congestion), which can reduce the time of execution of the problem. Olena Bakulich and Vitaliy Svatko proposed this work.Aleksandar Kaplar about improving a distributes agent Based ACO for solving TSP. In this paper, the execution speed of TSP using an agent and client based approach. Here an ant is presented as an agent and client as a destiny city. It is proved that with less number of messages good solution can be found. Milan Vidakovic, Nikola Luburic, an Mirjana Ivanovic proposed this work. LIU Shufen about Pheromone model selection in ACO for TSP. In this paper, they presented the different models of pheromones. It proves that by selecting a correct and good pheromone level gives the correct outcome and solution for the problem. It shows that an ideal pheromone needs large search space for solving the problem. LENG Huang, HAN Lu proposed this work.

\section{Applications of ant colony optimization}

\subsection{Traveling sales person}

In this problem sales person will given a set of cities to be visited, he should visit all the cities and return to starting point and every city should be visit only once this is a np-hard problem it has many solution, he should come up with best shortest path to visit all cities in less time.

\subsection{Load balancing in telephone networks}

When a new call is arriving to a number, routes of individual new call and checks that old calls expired or not load on nodes if it is nor expired new will fail to connect due to congestion updates the pheromone table and routes of ants,new ant will be launched only after old ants die in the same after ending the old calls new call will be connected.

\subsection{Ant net}

The calculation takes after an indistinguishable standards from that talked about as to phone systems. Ants scan for a base cost way interfacing the source and goal hubs. The decision of way is made by a comparative run the show. Ant net has been turned out to be exceptionally versatile and hearty under application to various systems and situations. It is viewed as being among the best in class directing calculations.

\subsection{Job shop scheduling}

Job shop scheduling is the one of the best combinatorial problem which it should assigned to resources are particular times suppose there are $\mathrm{n}$ jobs it should understand to which it should gives resources to complete the $\mathrm{n}$ jobs in time.

\subsection{Vehicle routing}

Vehicle directing issues (VRPs) [1]. These are issues where an arrangement of vehicles positioned at a terminal needs to serve an arrangement of clients before coming back to the stop, and the goal is to limit the quantity of vehicles utilized and the aggregate separation went by the vehicles.it is only augmentation of Traveling Sales Person.

\section{Travelling salesman problem}

\subsection{History}

The general type of the TSP gives off an impression of being have been first concentrated by mathematicians beginning in the 1930 s by karl Menger in Vienna and Harvard. The issue was later advanced by Hassler Whitney and Merrill Flood at Princeton. A definite treatment of the association amongst Menger and Whitney, and the development of the TSP as a subject of study can be found in Alexander Schrijver's paper "on the historical backdrop of combinatorial streamlining (till 1960)".

\subsection{Problem \& explanation}

The ant colony optimization is applies to traveling salesman problem. Starting form basic algorithm of ant colony on several changes in the algorithm this problem can be solved. Traveling sales man problem is well known NP-hard problem. By using artificial ant colony we can solve traveling salesman problem.

By using artificial colony we can generate ants that are capable of finding the shortest path by using the information given by the other with pheromone trail deposited on their path [1].

TSP is to find the shortest path for a salesman for a finite Number of urban communities. Businessperson is requested to begin from an irregular city by going by every city precisely once and after that to come back to the beginning city A total Weighted diagram $\mathrm{G}=(\mathrm{N}, \mathrm{E})$ [7] can be utilized to speak to a TSP, where $\mathrm{N}$ is the arrangement of $n$ urban areas and $E$ is the arrangement of edges (ways) completely interfacing all urban areas. TSP is additionally called the Hamiltonian circuit, which is a shut visit going to every city in $G$ precisely once. Each edge (I, j) has a place with $E$ is relegated a cost dij, which is the separation between urban areas I and $\mathrm{j}$. dij can be characterized in the Euclidean space and is given as takes after salesperson issue is outstanding NP-difficult issue. By utilizing counterfeit insect province we can take care of traveling sales representative issue [6].

By using artificial colony we can generate ants that are capable of finding the shortest path by using the information given by the other with pheromone trail deposited on their path.

TSP is to locate the most limited excursion of a sales representative for a limited number of urban communities. A salesperson is requested to begin from an arbitrary city by going by every city precisely once and after that to come back to the beginning city An entire Weighted chart $\mathrm{G}=(\mathrm{N}, \mathrm{E})$ can be utilized to speak to a TSP, where $\mathrm{N}$ is the arrangement of $\mathrm{n}$ urban communities and $\mathrm{E}$ is the arrangement of edges (ways) completely interfacing all urban communities. TSP is likewise called the Hamiltonian circuit, which is a shut visit going to every city in G precisely once. Each edge $(i, j)$ has a place with $E$ is appointed a cost dij, which is the separation between urban communities $i$ and $j$. dij can be characterized in the Euclidean space and is given as takes after.

$\mathrm{d} i \mathrm{j}=\operatorname{sqrt}$ of $(\mathrm{Xi}-\mathrm{Xj})^{2}+(\mathrm{Yi}-\mathrm{Yj})^{2}[1]$

Where (xi, yi) and (x j, y j) are the coordinates of city $i$ and $j$, respectively.

For symmetric TSP's, the length between any two cities are not dependent of the path of traversing the arcs, that is, $\mathrm{dij}=\mathrm{dji}$ for every two points .further in general asymmetric TSP for at least for 2 nodes that is $\mathrm{i}$; $\mathrm{j}$ we have .dij not equal to dji.. the inter-city distances are calculated using the Euclidean norm.

\section{Implementation}

Below flow chart is about step by step procedure of finding the shortest path in any problem ,but it may varies at parameters and conditions, and here we should give a certain time limit and $n$ number of ants and how many no of iterations should do. 


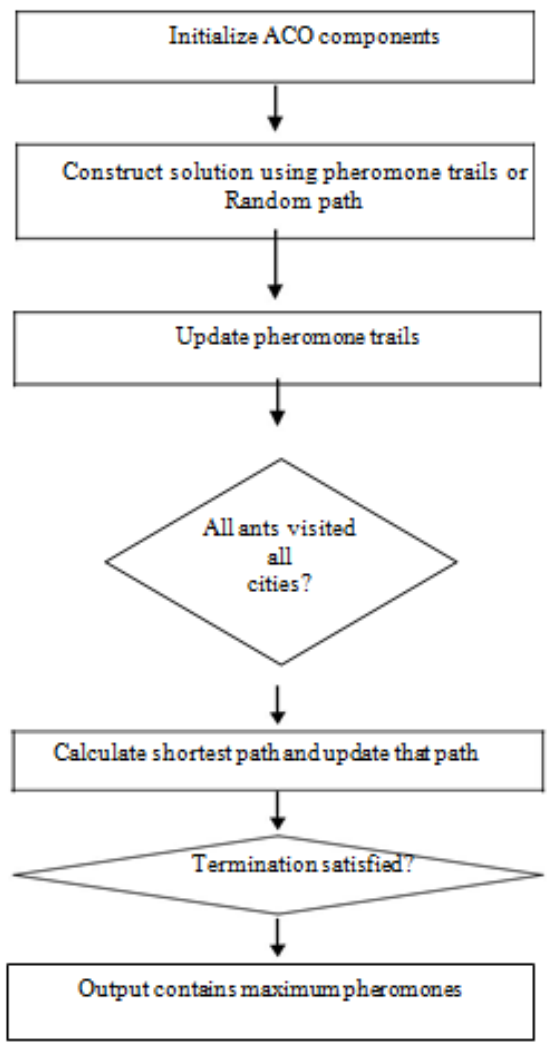

Fig. 1: Solving TSP Using ACO.

As the flow chart shown at first ant colony parameters will initialize in the step one, next step ants will start moving based on the pheromone trail or randomly and they will update the pheromone if cities are visited by all ants then we should compute the length of shortest path that is optimal path and if all ants didn't visit the all cites it should goes to the step 2 that going to another path until they visit all cites.

Algorithm 1: The Ant Colony Optimization (ACO)

Step 1: Initialize and set pheromone values;

Step 2: Repeat until ants find the optimal path do

Step 3: develop Ants solutions

Step 4: Apply local search for different paths

Step 5: Update pheromones

At first we should initialize parameters like how many ants and pheromones trail [7] and now ants will start at any point and goes in the different direction to find the food if the find the food they start lying the pheromones on all the way to nest that is the initial point, after if any ant finds the food in direction they to come to starting point(nest) while returning to find some more food they will see pheromones trail path they will decide this may be the shortest path or to find the food they will go in the direction and may not go but after sometime due to many ants goes in the direction that path will more pheromones and next ant will goes in that direction and all remaining ants will goes in that path if the finds the path while loop in the algorithm will terminate and displays the final path if does not again start for the searching different path by applying local search and the step is to update the pheromones without updating ants can't find the best path[4].

Algorithm 2: Traveling Sales Man Using Aco

Step1: Intitialize and set pheromones values;

Step2: Repeat until salesman visit all cities do

Step3: Visit all cities by following trails

Step4: Apply local search for different paths

Step5: Update pheromones

By using this algorithm [4] we are applying for traveling sale man problem like sale man starts at one point that is initial point and visit next city, visit all the cities which set in the starting step and without repeating the cities that is the condition and again it should comes to the initial point where he starts by visiting all cites like ants, while loop should execute until salesman visit all cities do the condition visit all remaining cities by following the trails and by applying local search to find the different path to visit cities and update pheromones.

\subsection{Tour construction}

At first, every ant is put on some haphazardly picked city. At every development step, insect $\mathrm{k}$ applies a probabilistic activity decision run the show. Specifically, the likelihood with which subterranean insect $k$, as of now at city I, goes to city $j$ at the $t$ th cycle of the calculation is:

$\left[T_{\mathrm{ij}}(\mathrm{t})\right]^{\alpha} \cdot\left[\eta_{\mathrm{ij}}\right]^{\beta}$

$\mathrm{P}_{\mathrm{ij}}^{\mathrm{k}}(\mathrm{t})=$ if $\mathrm{j} \in \mathrm{N}_{\mathrm{i}}^{\mathrm{K}}[4]$

$\sum_{\mathrm{IeNI}^{\mathrm{k}}}\left[\mathrm{T}_{\mathrm{il}}(\mathrm{t})\right]^{\alpha} \cdot\left[\eta_{\mathrm{il}}\right]^{\beta}$

where $\eta \mathrm{ij}=1 / \mathrm{dij}$ is a from the earlier accessible heuristic esteem $\alpha$ and $\beta$ are two parameters which decide the relative is the doable neighborhood of insect $\mathrm{k}$, that is, the arrangement of urban communities which subterranean insect $\mathrm{k}$ has not yet gone by. The part of the parameters and is the accompanying. In the event that $=0$, the nearest urban communities will probably be chosen. In the event that $=0$, just pheromone enhancement is grinding away. Inquiry stagnation is characterized in as the circumstance where every one of the ants take after a similar way and build a similar arrangement.

\subsection{Updating the pheromone}

After all ants have built their visits, the pheromone trails are refreshed. This is finished by first bringing down the pheromone quality on all circular segments by a steady factor and after that enabling every insect to include pheromone the curves it has gone to $\mathrm{m}$

$\operatorname{Tij}(t+1)=(1-\rho) \cdot \operatorname{Tij}(t)+\sum \mathrm{k}=1 \Delta \mathrm{T}_{\mathrm{ij}}(\mathrm{t})[1],[4]$

$\mathrm{K}=1$

Where $0<\rho \leq 1$ [7], [9] is the pheromone trail vanishing. The parameter $\rho$ is utilized to keep away from boundless amassing of the pheromone trails. On the off chance that a bend is not picked by the ants, its related pheromone quality diminishes exponentially. $\Delta \tau^{\mathrm{k}_{\mathrm{ij}}}(\mathrm{t})$ is the measure of pheromone subterranean insect $\mathrm{k}$ puts on the curves it has gone by.

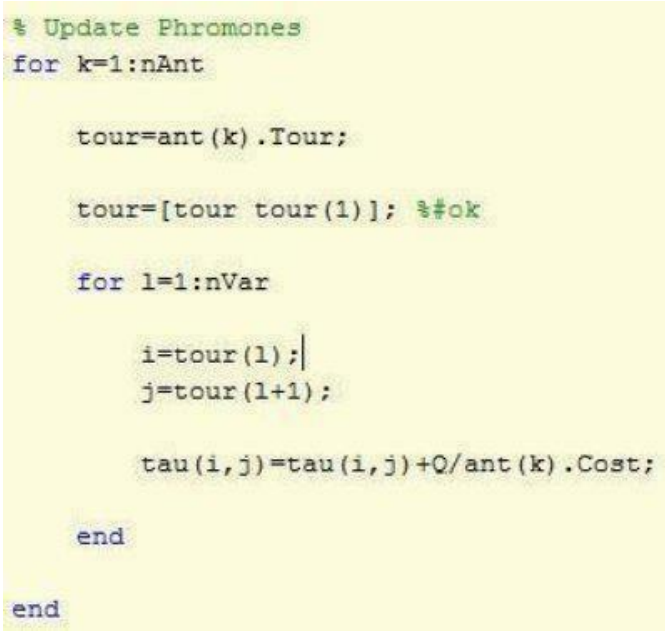

Fig. 2: Updating Pheromones.

We implemented same formula in mat lab for updating the pheromones For implementing we have taken 30 iterations and total no of ants are 4 for creating the graph that is on $\mathrm{x}$ and $\mathrm{y}$ axis for pointing the cities we taken the input values as 


\section{$X=\left[\begin{array}{lllllll}10 & 0 & 0 & 60 & 30 & 40 & 50\end{array}\right]$}

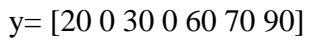

For finding distance using the formula,

$D(i j)=\operatorname{sqrt}\left(\left(x(i)-x(j)^{\wedge} 2+(y(i)-y(j))^{\wedge} 2\right)[9]\right.$

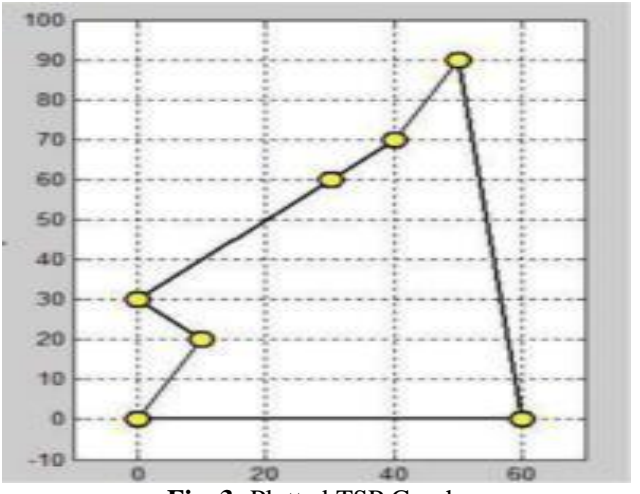

Fig. 3: Plotted TSP Graph.

The above graph is the final shortest path for the given input.

Table 1: Best Cost

\begin{tabular}{lll}
\hline ITERATION & 3 & BEST COST $=292.1325$ \\
\hline ITERATION & 4 & BEST COST $=265.9859$ \\
ITERATION & 5 & BEST COST $=265.9859$ \\
ITERATION & 6 & BEST COST $=265.9859$ \\
ITERATION & 7 & BEST COST $=265.9859$ \\
ITERATION & 8 & BEST COST $=265.9859$ \\
ITERATION & 9 & BEST COST $=265.9859$ \\
ITERATION & 10 & BEST COST $=265.9859$ \\
ITERATION & 11 & BEST COST $=265.9859$ \\
ITERATION & 12 & BEST COST $=265.9859$ \\
ITERATION & 13 & BEST COST $=265.9859$ \\
ITERATION & 14 & BEST COST $=265.9859$ \\
ITERATION & 15 & BEST COST $=265.9859$ \\
ITERATION & 16 & BEST COST $=265.9859$ \\
ITERATION & 17 & BEST COST $=265.9859$ \\
ITERATION & 18 & BEST COST $=265.9859$ \\
ITERATION & 19 & BEST COST $=265.9859$ \\
ITERATION & 20 & BEST COST $=265.9859$ \\
ITERATION & 21 & BEST COST $=265.9859$ \\
ITERATION & 22 & BEST COST $=265.9859$ \\
ITERATION & 23 & BEST COST $=265.9859$ \\
ITERATION & 24 & BEST COST $=265.9859$ \\
ITERATION & 25 & BEST COST $=265.9859$ \\
ITERATION & 26 & BEST COST $=265.9859$ \\
\hline
\end{tabular}

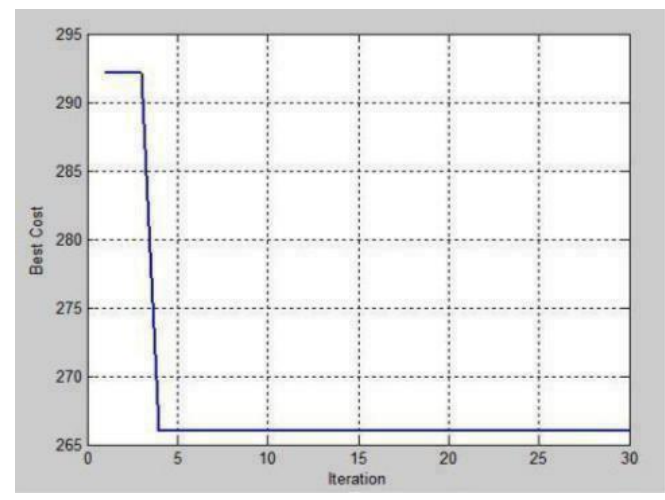

Fig. 4: Best Cost Result Graph.

This is the graph between the best cost and iterations for the above the best cost is the 265.9859 if you look at the iteration point at the iteration 4 best solution is found that is shortest ant remaining ants are following the same paths thats why in remaining iterations cost is same hence that is the best path .

\section{Applications of TSP}

\subsection{Boring of printed circuit sheets}

An immediate use of the TSP is in the boring issue of printed circuit sheets (PCBs).To associate a conductor on one layer with a conductor on another, gaps must be bored through the board. The gaps might be of various sizes, To penetrate two gaps of various breadths successively, the leader of the machine needs to move a tool kit and change the penetrating hardware. This is very tedious. Along these lines plainly one needs to pick a few breadth, penetrate all openings of a similar distance across, change the bore, bore the gaps of the following breadth, penetrate all openings of a similar distance across, change the bore, bore the gaps of the following Measurement, and so on. Along these lines, this penetrating issue can be seen as a progression of TSPs, one for each opening width, where the 'urban communities' are the underlying position and the arrangement of all openings that can be bored with one and a similar penetrate.

\subsection{Updating gas turbine motors}

It works when gas turbine motors of air ship must be updated. To ensure a uniform gas course through the turbines there are spout control vane gatherings situated at every turbine organize. Such a get together essentially comprises of various spout manage vanes joined about its outline. Every one of these vanes have singular attributes and the right arrangement of the vanes can bring about considerable advantages (decreasing vibration, expanding consistency of stream, lessening fuel utilization).

\subsection{X-ray crystallography}

Investigation of the structure of precious stones is an vital use of the TSP. Here a X-beam diffract meter is utilized to get information of the structure of crystalline objects. And it also estimate the power of $\mathrm{X}$ - beam impressions of the precious stone from different positions. Though the estimation itself can be achieved very quick, there is an impressive overhead in situating time up to many hundred places must be acknowledged for a few tests.

In the above illustrations we can allude to, the situating includes moving four engines. The time expected to move from one place to the another will done precisely. The consequence of the explore does not rely upon the succession in which the estimations at the different places are taken.

\subsection{Computer wiring}

Parts are on a PC motherboard and a different types of pins needs to associated. Rather than the standard situation where a Steiner tree association rule is needed, the prerequisite is that close to wires are joined to each pin.For every part we need a predetermined joining and exit points for this wiring. This issue likewise sums to taking care of a Hamilton way issue with the distinction that the separations are mostly asymmetric and beginning and ending points are indicated.

\subsection{Printing press scheduling problem}

One of the basic employments of the m TSP rises in arranging a press for a multi-renditions. There have a 5 arrangements of chambers between which the paper moves and the both sides of a page are printed in the meantime. There are 3 kind of structures, specifically 2-, 4-and 6-page outlines, which are used to print the discharges. The arranging issue involves picking which point will be starting point and the length of each run. In the m TSP vocabulary, it change costs from city to city. 


\section{Conclusion}

In this paper we showed a way to find the shortest path by using Ant colony optimization (ACO) by taking the example of TSP (travelling salesman problem). As a result, by following the ACO we can find optimal solution in short time not only the TSP we can solve many np-hard problems by ACO and also showed various recent applications of TSP.

\section{References}

[1] Dweepna Garg and Saurabh Shah "Ant colony optimization for solving traveling salesman problem" vol 5, no 1,January-june 2011

[2] Potyin J.Y (1993), The Traveling salesman problem: A Neural Network Perspective ORSA Journal of computing, 5 (4), 328-347. https://doi.org/10.1287/ijoc.5.4.328.

[3] "Ant colony System. A cooperative learning approach to the traveling salesman problem,"IEE transaction on Evolutionary computatiom, vol 1, no-1, pp 53-66, 1997.

[4] M. Dorigo and G. Di caro,'The Ant colony Optimization metaheuristic", in new in optimization, D.corneerl. eds., Mcgraw Hill, London, UK, pp. 11-32, 1999.

[5] A.I \& Kennington, J. L (1986).the asymmetric m-traveling salesman problem, a duality based branch and bound algorithm, Discrete Applied Mathematics, vol NO,13,pp-259-76.

[6] Lin, S. (1986)"computer solution of the traveling salesman problem", Bell syst journal 44, 2245-2269. https://doi.org/10.1002/j.1538-7305.1965.tb04146.x.

[7] Dorigo, M \& Gambardella, (1997)"Ant colonies for the traveling salesman problem"Biosystemsm43, 73-81. https://doi.org/10.1016/S0303-2647(97)01708-5.

[8] "ACO algorithm with guaranteed convergence to the optimal solution", Information Processing Letters, vol, 82, no.3, pp 145-153, 2002. https://doi.org/10.1016/S0020-0190(01)00258-7.

[9] Gao Shang Zhang Lei Zhuang fengting Zhang Chunxian School of electronics and information Jianguru University of science and technology, Zhenjiang 212003, china.

[10] C.S Jeong nad M.H Kim, "Fast parallel simulated annealing for traveling salesman problem", Neural Network, vol.3 (1990), pp.947-953

[11] JinhuiYang,Xiaohushi,MaurizioMarchese and sanctum Liang Yanchum Liang, "Ant colony optimization for generalized TSP problem", college of computer science Jilin university,changchum 130012,china.

[12] Zalilah Abd Aziz, "Ant colony Hyper-heuristics for Travelling Salesman Problem", Mara University,2015 IEEE\{IRIS 2015\}

[13] Michalis Mavrobouniotis Felipe M.Muller Shengxiang yang, "Ant colony optimization with local search for Dynamic Travelling salesman problem: IEEE, vol.47 no. 7, july 2017.

[14] Hassan Ismkhan,"Effective heuristics for ant colony optimization to handle large scale problem", swarm and evolutionary computation 32(2017)140-149

[15] Srikanta parnaik,"Route optimization by Ant colony optimization technique", school of study on computer science \& IT, Raipur(Chhattisgarh)492010,India

[16] Mateuz sekara Michal Kowalski,Aleksandar Byrski and bipin indurkhya,"Multi-pheromone ant colony optimization for sociocognitive simulation process".vol.51,2015.

[17] Chi-bin cheng, chun-pin mao," A modified ant colony system for solving he traveling salesman problems", mathematical and computer modelling 46(2007).

[18] Viktor danchuk, oelna bakulich, vitaliy syatko,"An improvement in ant algorithm method for optimizing a transport route with regard to raffix flow", National transport university, Ukraine.

[19] Aleksandar kaplar milan vidakovic,nikola luburic and mirjana ivanovic,"improving a distributed agent based ant colony optimization for solving traveling salesman problem", university of novi sad, Serbia.

[20] LIU shufen Lenf Huang and hanlu,"Pheromone model selection in ant colony optimization for the traveling salesman problem",jilin university, vol 26,No 2,13002,china 